\title{
Biparametric investigation of the general standard map: multistability and global bifurcations
}

\author{
Priscilla A. Sousa-Silva ${ }^{1}$ (D) Maisa O. Terra ${ }^{2}$
}

Received: 20 February 2017 / Revised: 25 October 2017 / Accepted: 21 November 2017 /

Published online: 29 November 2017

(C) SBMAC - Sociedade Brasileira de Matemática Aplicada e Computacional 2017

\begin{abstract}
We investigate multistability and global bifurcations in the general standard map, a biparametric two-dimensional map. Departing from the conservative case of the map, we describe the evolution of periodic solutions and their basins of attraction as dissipation builds up, paying special attention on how the biparametric variation affects multistability. We examine general and specific phenomena and behavior for three distinct dynamical regimes, namely small, moderate, and large damping and different forcing amplitudes. Also, we report numerically the mechanism of global bifurcations associated to small chaotic attractors in the multistable system. Several global bifurcations are investigated as dissipation increases. Specifically, through the characterization of an interior, a merging and a boundary crisis, we study the crucial role played by fundamental hyperbolic invariant structures, such as unstable periodic orbits and their stable and unstable invariant manifolds, in the mechanisms by which the phase space is globally transformed.
\end{abstract}

Keywords General standard map · Crises · Global bifurcations · Unstable periodic orbits and manifolds · Multistability

Mathematics Subject Classification 37M99 · 37N99 - 37E99

Communicated by Jorge X. Velasco.

$凶$ Priscilla A. Sousa-Silva

priscilla.silva@sjbv.unesp.br

Maisa O. Terra

maisa@ita.br

1 UNESP, Universidade Estatual Paulista, Câmpus de São João da Boa Vista, Av. Professora Isette Corrêa Fontão, 505, São João da Boa Vista, SP, Brazil

2 Instituto Tecnológico de Aeronáutica, Praça Marechal Eduardo Gomes 50, São José dos Campos, SP, Brazil

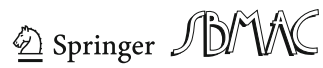




\section{Introduction}

The general standard map derives from a model for the motion of a periodically kicked mechanical pendulum or kicked rotator, with the inclusion of damping. This mechanical system consists of a bar attached at one end to a pivot and subjected to a vertical periodic impulsive force acting on the other end of the bar (Ott 1993). The discrete time system

$$
\begin{aligned}
& \theta_{k+1}=\theta_{k}+p_{k}(\bmod 2 \pi) \\
& p_{k+1}=(1-v) p_{k}+f_{0} \sin \left(\theta_{k}+p_{k}\right)
\end{aligned}
$$

derives from the Poincaré Map related to the state of the rotator after each periodic kick.

In Eq. (1), $\theta$ and $p$ are the angular position and the momentum of the rotator, and $f_{0} \geqslant 0$ and $v \in[0,1]$ are real parameters that account for, respectively, the kick amplitude, that is, the forcing applied in the continuous time system at times $k T, k=1,2, \ldots$, with $T$ as a constant period, and the viscous damping. This dynamical system presents a symmetry given by $(\theta, p) \rightarrow(-\theta,-p)$.

When $v=0$ the pivot is frictionless and the correspondent area-preserving map is known as the Chirikov-Taylor map or the Chirikov standard map, a paradigmatic model for two degrees of freedom Hamiltonian systems (Chirikov 1979). When $v=1$, Eq. (1) reduces to the one-dimensional circle map with zero rotation number.

Several continuous-time dynamical systems and maps can be locally reduced to the standard map or to its general version that includes dissipation. So, the understanding and analysis of the diversity of dynamical phenomena present in this dynamical system has great relevance. This mathematical model has applications in the particle dynamics in magnetic traps and accelerators, atoms dynamics in a periodic potential, comet dynamics in the Solar system, and electron magnetotransport in a resonant tunneling diode, among others.

Many aspects and dynamical phenomena can be examined using the area-preserving and dissipative standard map. Among them we can mention the investigation of the breakdown and analytic structure of invariant curves and solutions (Berretti and Chierchia 1990; Calleja and Celletti 2010); collision of invariant bundles of quasi-periodic attractors in dissipative regime (Calleja and Figueras 2012); out-of-equilibrium states and Tsallis statistics (Tirnakli and Borges 2016); periodic, quasi-periodic, and general solutions (Celletti et al. 1998; Celletti and Di Ruzza 2011); and separatrix splitting and diffusive processes (Gelfreich 1999; Miguel et al. 2013, 2015). Notwithstanding the amount of literature regarding particular aspects of this model, few contributions investigate both dynamical regimes of the mathematical model in order to inspect dynamical phenomena from conservative to dissipative cases and to examine the role played by fundamental invariant solutions of the system.

In this work, we perform a numerical biparametric investigation of the general standard map. Starting with the conservative case, we describe the evolution of periodic solutions and their basins of attraction as dissipation builds up and investigate how the parameters affect the property of multistability. Also, we characterize three different global bifurcations related to chaotic attractors of the multistable system, namely an interior crisis, a merging crisis, and a boundary crisis. By identifying the dynamical elements that take part in these crises, we illustrate the crucial role played by fundamental invariant structures, specifically, unstable periodic orbits (UPOs) and their hyperbolic invariant manifolds, in the mechanisms by which the phase space is globally transformed.

The paper is organized as follows: Section 2 presents a brief review of the conservative case. Section 3 deals with the biparametric bifurcations of asymptotic solutions in the dissipative case, inspecting some properties of the basins of attraction and discussing the multistability properties in three remarkably distinct dynamical regimes: small, moderate 
and large damping. Also, we consider at least three magnitudes of the forcing parameter and show how solutions on the phase space depends on them. In Sect. 4, three different global bifurcations are characterized. Finally, Sect. 5 presents the final remarks and conclusions.

\section{Highlights of the conservative case}

We start by recalling the typical and well-known dynamics of the area-preserving standard map $(v=0)$. In this case,

$$
\operatorname{det}\left[\begin{array}{ll}
\frac{\partial \theta_{k+1}}{\partial \theta_{k}} & \frac{\partial \theta_{k+1}}{\partial p_{k}} \\
\frac{\partial p_{k+1}}{\partial \theta_{k}} & \frac{\partial p_{k+1}}{\partial p_{k}}
\end{array}\right]=\operatorname{det}\left[\begin{array}{cc}
1 & 1 \\
f_{0} \cos \left(\theta_{k}+p_{k}\right) & 1+f_{0} \cos \left(\theta_{k}+p_{k}\right)
\end{array}\right]=1,
$$

and the phase space reduces to the surface of a torus by taking the angular momentum modulo $2 \pi$. Although this representation is convenient for displaying the conservative structure of the phase space, $p$ and $p+2 \pi k, k \in \mathbb{Z}$, are not physically equivalent, given that, in general, they correspond to different values of the kinetic energy of the rotator.

In the conservative case, the dynamics is ruled by the characteristic phenomenology of Hamiltonian systems and depends exclusively on the forcing parameter. For $f_{0}=0$, the system is integrable and $p=p_{0}$ is a constant of the motion. Solutions are quasi-periodic (if $p_{0} / 2 \pi$ is an irrational number) or periodic (if the ratio $p_{0} / 2 \pi$ is rational) or static (if $p$ is zero). When $f_{0}>0$, integrability is broken and the phase space contains islands of stability associated with KAM surfaces and chaotic seas lying between the KAM curves. As $f_{0}$ increases, the tori of the unperturbed system are destroyed or deformed, according to KAM theorem and as more tori are destroyed, the chaotic regions spread through the phase space.

Figure 1 shows the phase space of the conservative standard map for different values of the forcing parameter. In Fig. 1a, corresponding to $f_{0}=0.5$, the KAM tori, which are deformed remnants of the non-resonant tori that exist for $f_{0}=0$, prevent the spreading of the chaotic layers and restrict the kinetic energy of the rotator. Also, several stability islands can be seen around the Poincaré-Birkhoff solutions that originated from the resonant tori of the unperturbed system. The last torus encircling the cylinder $(\theta, p)$ disappears at $f_{0}^{c} \approx 0.971635 \ldots$ (Greene 1979) and the energy of the rotator can increase without bound. As $f_{0}$ increases, the deformed tori are destroyed, being replaced by chaotic regions with interspersed island chains, as seen in Fig. 1b, corresponding to $f_{0}=1.0$.

Increasing $f_{0}$ even further the island chains disappear while the chaotic region spreads, as illustrated in Fig. 1c, d, corresponding to $f_{0}=1.5$ and $f_{0}=2.5$, respectively. Finally, only a period- 2 island is discernible for $f_{0}=4.5$, as seen in Fig. 1e, and as $f_{0}$ increases, e.g., as with $f_{0}=7.5$ shown in Fig. $1 \mathrm{f}$, the island becomes smaller, until it is no longer discernible. For values of $f_{0}$ even larger, e.g., $f_{0}=8 \frac{8}{9}$, Chirikov (1979) found numerically that the entire torus appears to be, within available numerical resolution, ergodically densely covered by a single chaotic orbit.

Besides illustrating how the phase space changes as the forcing parameter varies, the values of $f_{0}$ of Fig. 1 were selected to match the investigation of the dissipative case of the following sections, providing a clear counterpart between the conservative and the dissipative dynamics.

Sandwiched between the preserved KAM tori, the hyperbolic solutions - created as the resonant tori are destroyed-account for the chaotic dynamics. The fixed point at the origin 

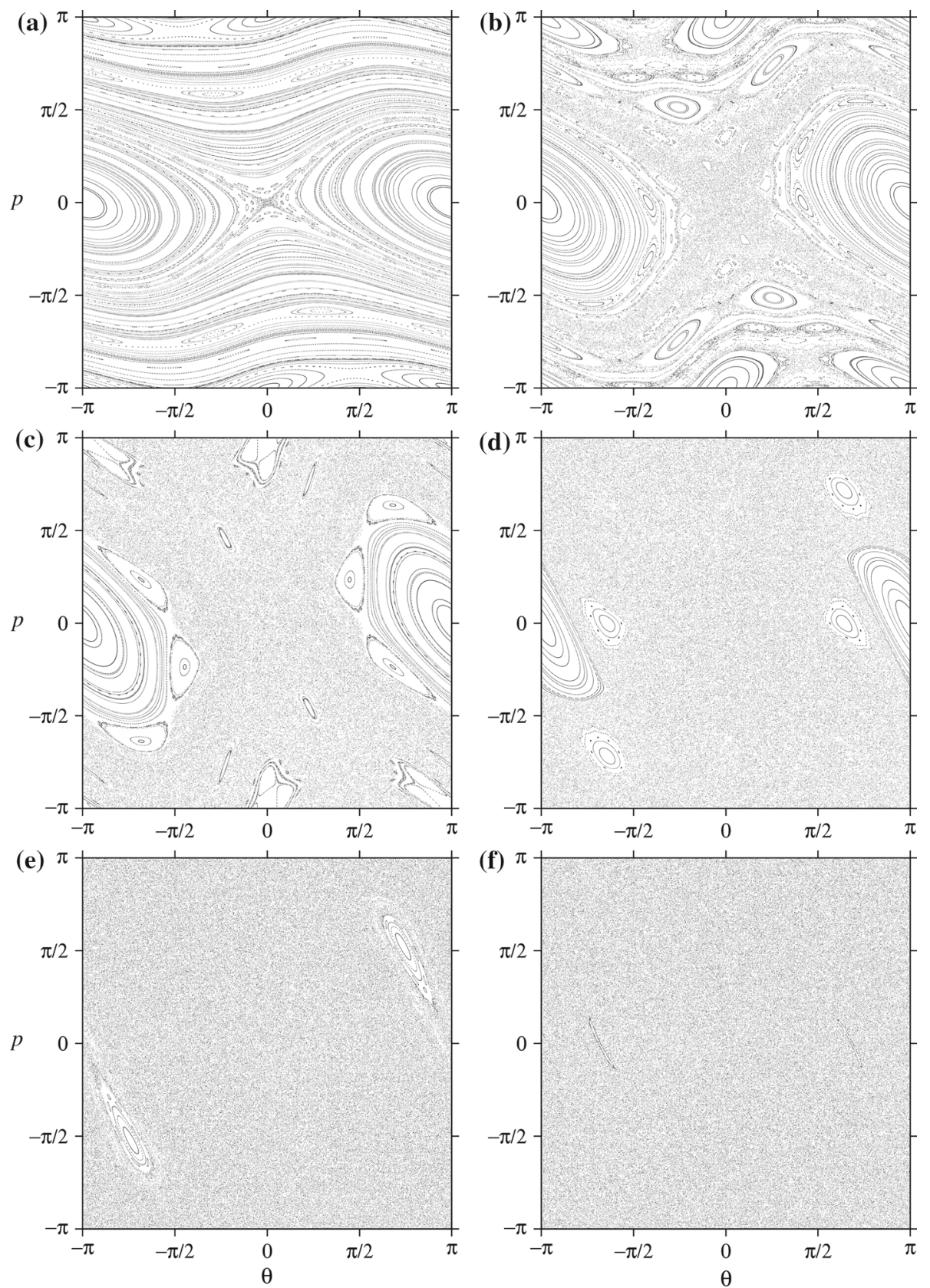

Fig. 1 Phase space of the standard map for a $f_{0}=0.5$, b $f_{0}=1.0, \mathbf{c} f_{0}=1.5, \mathbf{d} f_{0}=2.5$, e $f_{0}=4.5$, and f $f_{0}=7.5$, with $v=0$ 

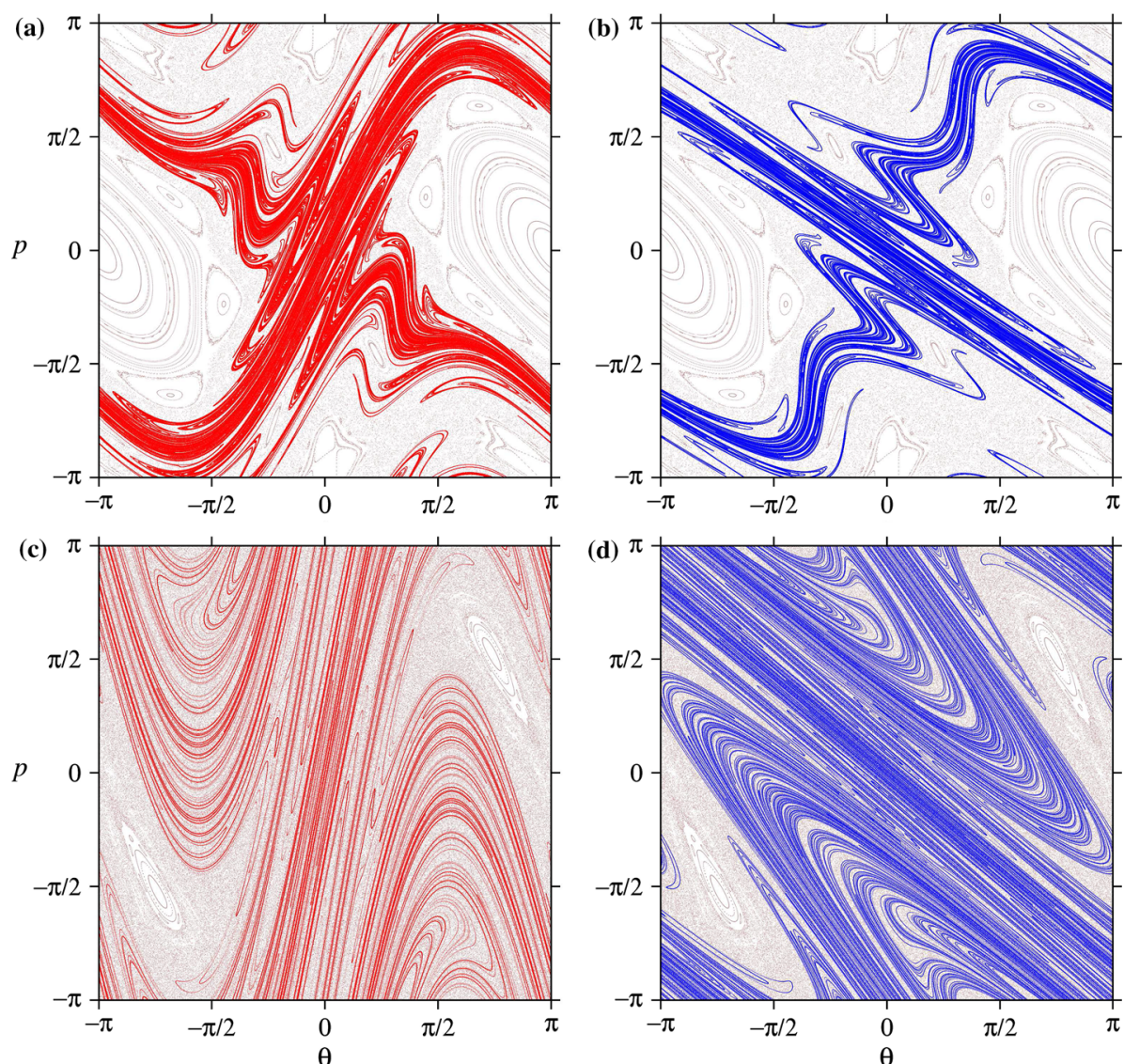

Fig. 2 a Unstable and $\mathbf{b}$ stable manifolds of the fixed point at the origin for $f_{0}=1.5$ and $v=0$. c Unstable and $\mathbf{d}$ stable manifolds of the fixed point at the origin for $f_{0}=4.5$ and $v=0$

is an example of that kind of solution. As expected from the Poincaré-Birkhoff theorem, the stability analysis gives that $(\theta, p)=(0,0)$ is saddle point for all $f_{0}>0, v=0$, with eigenvalues given by $\lambda_{1,2}=\frac{1}{2}\left(2+f_{0} \pm\left[f_{0}^{2}+4 f_{0}\right]^{1 / 2}\right)$, such that $\lambda_{2}=1 / \lambda_{1}$, with $\lambda_{1}>1$ for any $f_{0}>0$.

Figure 2 displays how the spatial disposition of the hyperbolic invariant manifolds of this static solution changes with $f_{0}$. Fig. $2 \mathrm{a}$, b illustrates the spatial disposition of, respectively, the unstable and the stable manifolds of the origin for $f_{0}=1.5$, while Fig. $2 \mathrm{c}$, d, shows the hyperbolic manifolds in the case of $f_{0}=4.5$. The disposition of the manifolds in the conservative case also affects the onset of the dynamical behavior when dissipation is introduced.

\section{The dissipative case: multistability and bifurcation structure}

When damping is taken into account, the variable $p$ cannot be taken modulo $2 \pi$, and the phase space corresponds to an infinite cylinder. The Jacobian of Eq. (1) is no longer unitary and is given by $(1-v)$. Moreover, the elliptic periodic orbits of the conservative system 
become sinks and the chaotic motion is replaced by long chaotic transients until the trajectory eventually settles down in one of the sinks (Feudel et al. 1996).

Applying the stationary condition to Eq. (1), we obtain that fixed points satisfy $p=$ $2 \pi m, m \in \mathbb{Z}$, and that $-1 \leqslant\left(2 \pi m \nu / f_{0}\right) \leqslant 1$, so that $|p| \leqslant f_{0} / v$. This means that the existence of fixed points is restricted to a cylindrical region of the phase space given by $[-\pi, \pi] \times\left[-f_{0} / \nu, f_{0} / \nu\right]$, called trapping region.

In particular, for a pair of $f_{0}>0$ and $v \in(0,1)$, the fixed points are given explicitly by

$$
\left(\theta^{*}, p^{*}\right)=\left(\arcsin \left(\frac{2 \pi m v}{f_{0}}\right), 2 \pi m\right), \quad m=0, \pm 1, \pm 2, \ldots,
$$

with $m<f_{0} / 2 \pi v$.

For each $m \neq 0$, Eq. (3) defines two static solutions, one stable and one unstable. Furthermore, for each $m>0$, each fixed point has a symmetric counterpart, such that $\left(\theta^{*}, p^{*}\right)$ corresponds to another fixed point $\left(-\theta^{*},-p^{*}\right)$ with $m<0$ and the same stability. On the other hand, for $m=0$, there are two fixed points: the origin, which is always unstable, and $( \pm \pi, 0)$ which is attractive for $v \in(0,1)$.

For each fixed point, the eigenvalues are given by

$$
\lambda_{1,2}=\frac{1}{2}\left\{\gamma \pm \sqrt{\gamma^{2}-4(1-v)}\right\},
$$

with $\gamma=2-v+f_{0} \cos \left(\theta^{*}+p^{*}\right)$.

Three main different scenarios can be identified when the value of the damping parameter increases for a fixed value of the forcing parameter $f_{0}>0$. Namely, for very small damping there exists a multitude of coexisting periodic attractors; for moderate damping, several multibanded small chaotic attractors also coexist with the periodic attractors; for large values of the damping parameter, a large chaotic attractor coexists with a few periodic attractors.

Indeed, Feudel et al. (1996) counted over 100 low period attractors coexisting for $v=0.02$ and $f_{0}=4.0$, and stated that the number of attractors can be arbitrarily high as $v \rightarrow 0$ with $f_{0} \in[0,5]$. So, multistability is the most prominent feature of the system for very small dissipation. This regime is illustrated by Figs. 3 and 4, which display the multiple asymptotic states for $v=0.025$ and $f_{0}=1.5$, and $f_{0}=4.5$, respectively. Using a grid of $10^{5}$ initial conditions uniformly distributed in the trapping region, we counted 32 attractors with 6 different periods in the first case and 94 attractors with 12 different periods in the second case. In agreement with Feudel et al. (1996), as $v$ decreases, the number of attractors increases. Also, attractors with higher period are found for the same resolution of the grid of initial conditions when $f_{0}$ increases. The basins of attraction of these periodic attractors are closely interwoven and appear to have fractal boundaries, resulting in an extremely low predictability of the final state for a given initial condition (Feudel et al. 1996; Martins and Gallas 2008).

The complexity of the bifurcation structure depends strongly on $f_{0}$ and $v$, as seen in the bifurcation diagrams for different values of $f_{0}$ of Fig. 5. In particular, for small $v$ and low to moderate $f_{0}$, the dynamical behavior is dominated by the appearance and disappearance of periodic attractors of several periods. On the other hand, chaotic attractors appear only as $f_{0}$ and $v$ increase. Indeed, chaotic attractors are expected to be rare because their basins shrink exponentially as $v \rightarrow 0$ and the intervals in parameter space in which they exist shrink geometrically with the Feigenbaum constant (Feudel and Grebogi 2003).

As an example, Fig. 5a, b shows the bifurcation diagram for $f_{0}=1.5$ obtained by following the asymptotic solutions at $v=0.025$ as $v$ decreases to zero (Fig. 5a), and as $v$ increases to 1.0 (Fig. 5b). That is, starting from the periodic asymptotic solutions that exist 


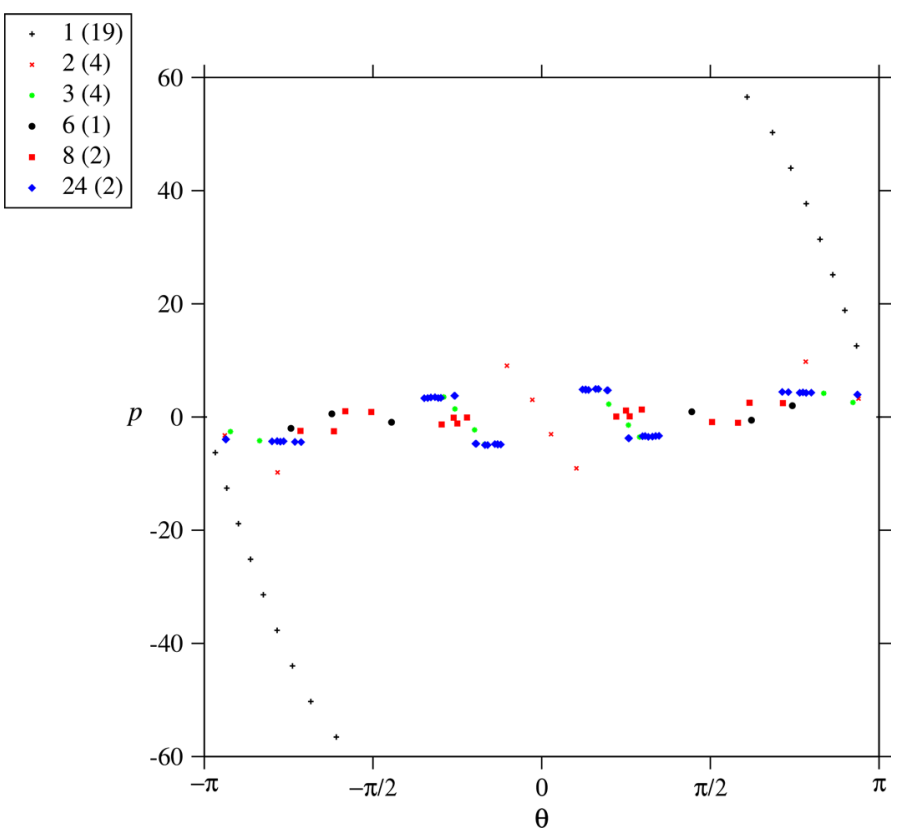

Fig. 3 Multiple periodic asymptotic states for $v=0.025$ and $f_{0}=1.5$

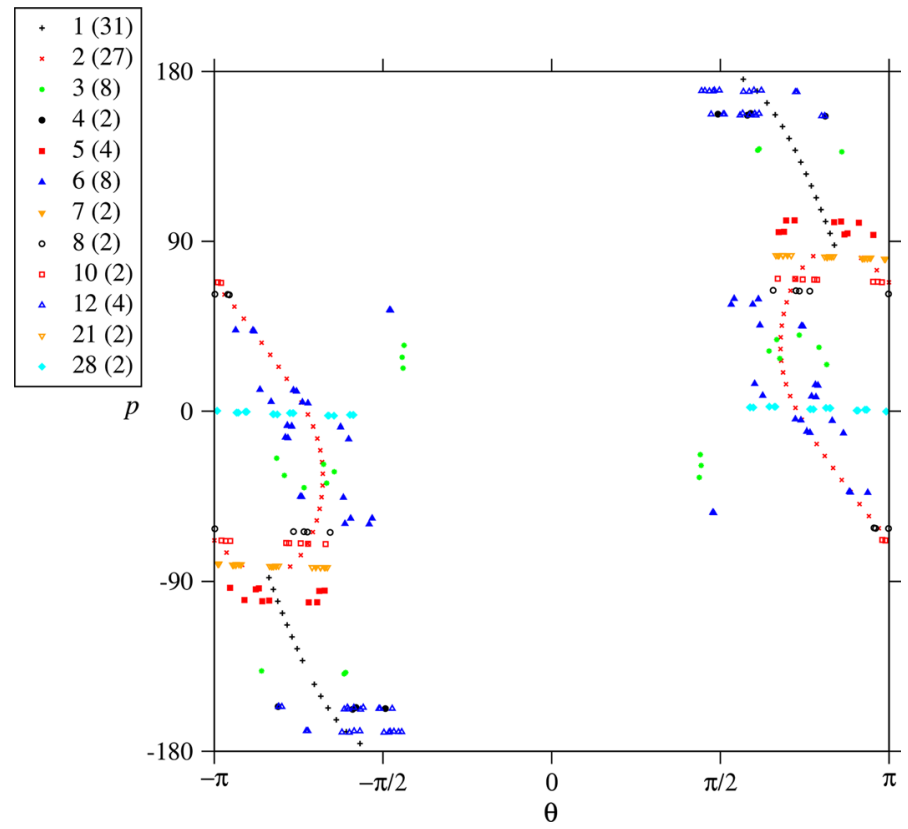

Fig. 4 Multiple periodic asymptotic states for $v=0.025$ and $f_{0}=4.5$ 

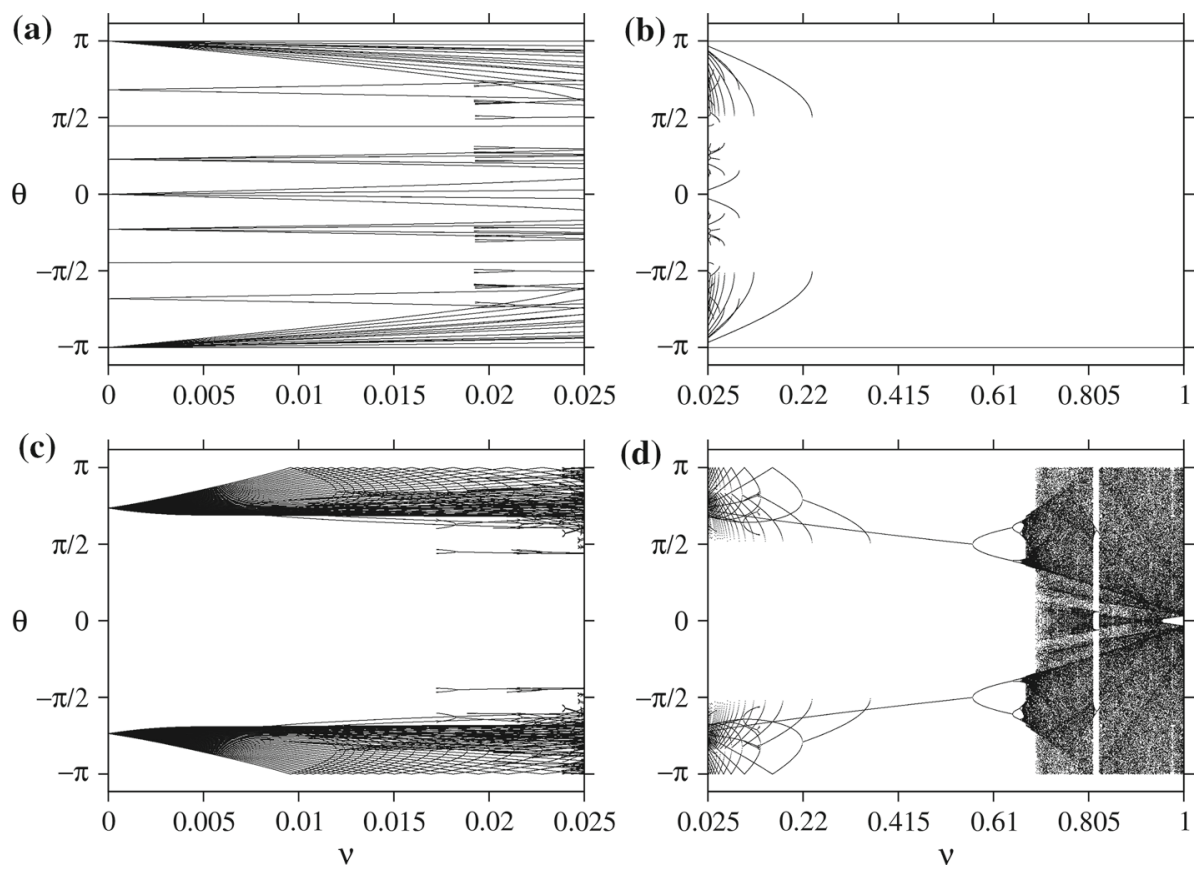

Fig. 5 Bifurcation diagrams given as a function of $v$. a $f_{0}=1.5,0<v \leqslant 0.025$; $\mathbf{b} f_{0}=1.5,0.025 \leqslant v \leqslant 1$; c $f_{0}=4.5,0<v \leqslant 0.025 ; \mathbf{d} f_{0}=4.5,0.025 \leqslant v \leqslant 1$

at $f_{0}=1.5, v=0.025$ (shown in Fig. 3), the map is iterated for every new value of $v$ using the final state of the previous value of $v$ as the initial condition. Then, the transient states are discarded, so only asymptotic states of the map are plotted in the diagrams. Also, only the asymptotic solutions related to those that exist for $f_{0}=1.5, v=0.025$ appear in the diagrams. Additionally, Fig. 5c, d shows the bifurcation diagram for $f_{0}=4.5$, starting from the periodic solutions at $v=0.025$ (those shown in Fig. 4) as $v$ decreases to zero (Fig. 5c), and as $v$ increases to 1.0 (Fig. $5 \mathrm{~d}$ ), with the same procedure described for the previous example.

As we reach moderate dissipation and low forcing, the typical behavior is the coexistence of a small number of low periodic attractors. For example, for $v=0.07$ and $f_{0}=1.5$, the stable fixed point at $( \pm \pi, 0)$, which from now on will be denoted by $P_{0}^{1}$, coexists with three pairs of period-1 attractors $P_{m}^{1}, m= \pm 1, \pm 2, \pm 3$, indicated by black $\times$ 's in Fig. 6, and two pairs of period- 2 attractors $P_{m}^{2}, m= \pm 1$, indicated by + 's.

In Fig. 6, the three pairs of symmetric fixed points and the two pairs of period-2 solutions are shown along with their basins of attraction. The white area corresponds to the basin of attraction of $P_{0}^{1}$.

All these attractors can be followed as $v \rightarrow 0$, where they correspond to center solutions. The stable manifolds of the saddle solutions that pair up with the center solutions in the Hamiltonian case, according to the Poincaré-Birkhoff theorem, relate to the boundaries of the basins of attraction of each attractor in the dissipative case.

In general, the symmetric pair of fixed points $P_{m}^{1}, \pm 1, \pm 2, \ldots$, disappear at a saddlenode bifurcation when $v / f_{0}=2 \pi|m|$ is reached. This is the critical value of parameter ratio in which the attractors reach the border of the trapping region. As an example of this process, Fig. 7 illustrates how the disposition of asymptotic solutions and their basins changes as $v$ 


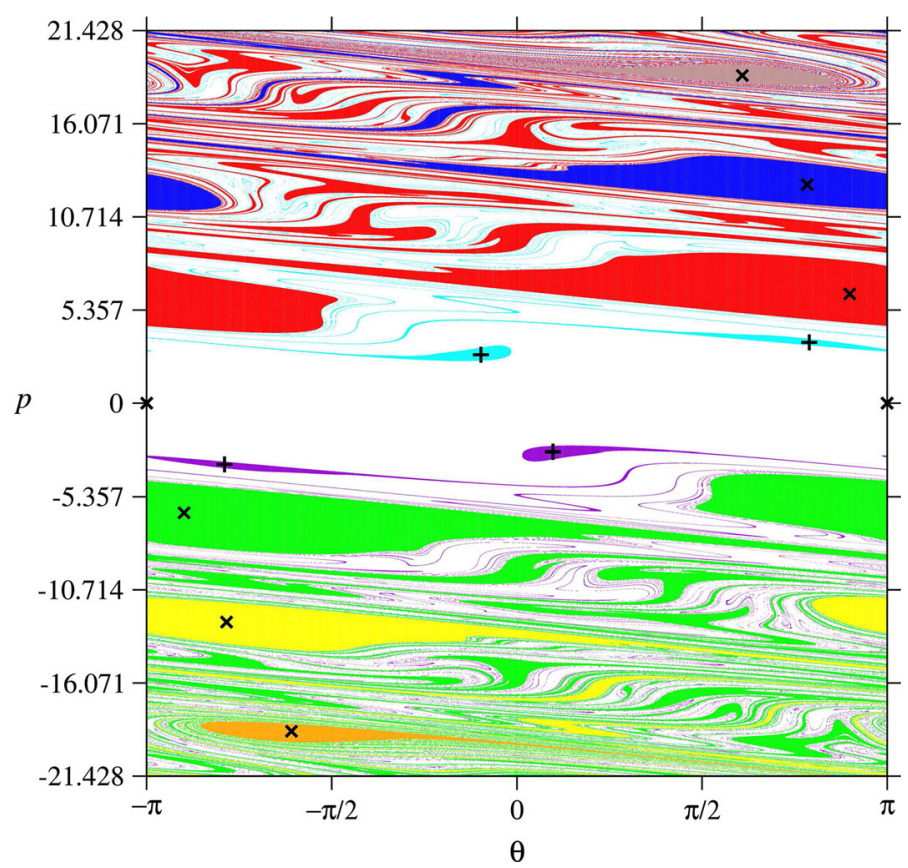

Fig. 6 Attracting periodic solutions and their basins of attraction for $v=0.07$ and $f_{0}=1.5$. Period- 1 stable solutions are depicted by $\times$ 's, while + 's indicate asymptotic period- 2 orbits

increases. The three period-1 attractors $P_{0}^{1}, P_{-1}^{1}$, and $P_{1}^{1}$ are shown along with their basins of attraction for $f_{0}=1.5$, and increasing $v$, namely $v=0.12$ (Fig. 7a), $v=0.156$ (Fig. 7b), $v=0.192$ (Fig. 7c), and $v=0.23$ (Fig. 7d), as $P_{-1}^{1}$ and $P_{1}^{1}$ approach the saddle-node bifurcation point at $\nu_{S N}=f_{0} / 2 \pi \approx 0.23873241$.

Moving on to the case of moderate dissipation and moderate forcing, the basins of attraction of small chaotic attractors become large enough as to allow that these asymptotic solutions are easily found throughout phase space. Generally the small chaotic attractors, which appear through period doubling cascades, coexist with periodic stable solutions with low period, as seen in Fig. 8, where a small chaotic attractor and three period-2 stable orbits, indicated by $\times$ 's, are shown for $f_{0}=4.5$ and $v=0.47330$. Figure 9 shows the basins of attraction of the periodic attractors and of the chaotic attractor shown in Fig. 8. Typically, these small chaotic attractors have basins of attraction with fractal boundaries.

In order to quantify the fractality of the sets we compute the uncertainty dimension $D_{0}$ of the fractal set embedded into a given set of initial conditions in the phase space, as described in Aguirre et al. (2001) and de Assis and Terra (2014). Let us consider a large number of randomly chosen initial conditions $\left(\approx 10^{4}\right.$ or more) on a line with constant $p=p_{0}$ in the two-dimensional phase space. We determine to which basin of attraction each initial condition $\left(\theta_{0}, p_{0}\right)$ belongs to. Then, we do the same for the initial conditions $\left(\theta_{0}, p_{0}-\epsilon\right)$ and $\left(\theta_{0}, p_{0}+\epsilon\right)$ for a small real $\epsilon$. If all three initial conditions belong to the same basin, $\left(\theta_{0}, p_{0}\right)$ is said to be certain; otherwise, $\left(\theta_{0}, p_{0}\right)$ is said to be uncertain. After all the initial conditions on the line are classified, we compute the fraction $f(\epsilon)$ of initial conditions that lead to uncertain final states and the uncertainty exponent $\alpha$ defined by $f(\epsilon) \propto \epsilon^{\alpha}$. Plotting $\ln f(\epsilon)$ vs. $\ln \epsilon$ for several values of $\epsilon$, we find $\alpha$, which corresponds to the slope of the line 

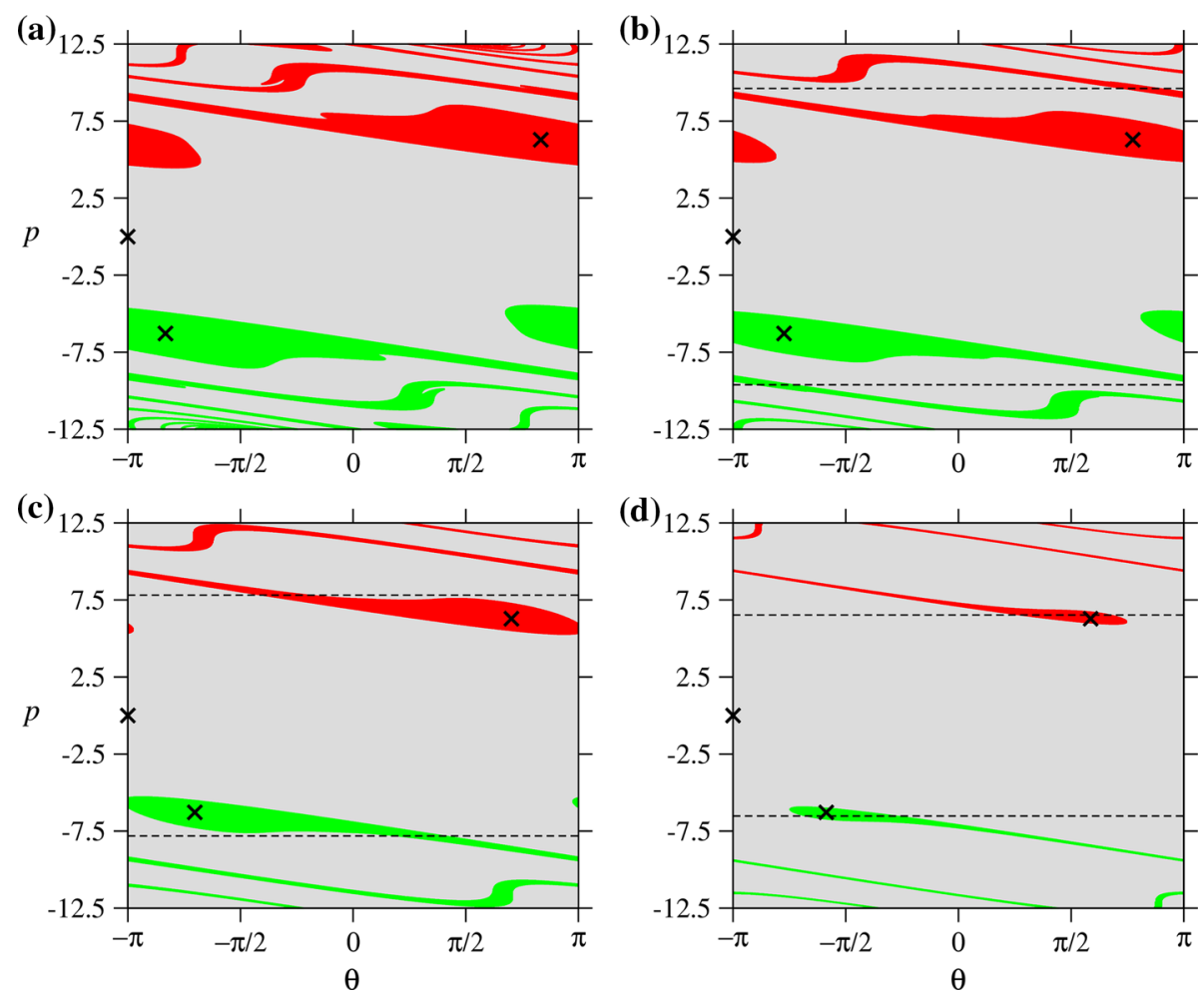

Fig. 7 The three attractive fixed points $P_{m}^{1}, m=-1,0,1$, indicated by $\times$, along with their basins of attraction for $f_{0}=1.5$, with $\mathbf{a} v=0.12$ ), $\mathbf{b} v=0.156$, $\mathbf{c} v=0.192$, and $\mathbf{d} v=0.23$. The dashed black lines delimit the trapping region in the phase space

in the range of $\epsilon$ for which the variation of $f(\epsilon)$ is linear. The fractal dimension $D_{0}$ of the fractal set embedded in the set of initial conditions is obtained by the relation $\alpha=D-D_{0}$, where $D$ is the dimension of the set of initial conditions. Finally, the dimension of the basin boundary is found by $D_{f}=D_{0}+N-1$, where $N$ is the dimension of the phase space, which equals two in the case of the standard map. In the example shown in Fig. 9, we computed $D_{0}$ for several lines with different values of $p_{0}$, resulting in a mean value of $D_{f}=1.92$ for the fractal dimension of the basin boundaries. The same approximate mean value for the fractal dimension is found by considering sets of initial conditions in line with constant $\theta_{0}$ and taking $\left(\theta_{0}-\epsilon, p_{0}\right)$ and $\left(\theta_{0}+\epsilon, p_{0}\right)$.

Besides the fractal dimension, we also quantify the fraction of points in each basin of attraction in relation to the total number of inspected initial conditions (ICs) for the basins shown in Fig. 9. Despite the high value of the fractal dimension of the basin boundary, we observe a large difference in these fractions. The basin shown on Fig. 9a, associated with the periodic attractor with $|p|<4$ and labeled by $P_{1}^{2}$, adds up $47.6 \%$ of the total ICs. On the other hand, the basins of attraction of the other period-2 attractors, $P_{+2}^{2}$ and $P_{-2}^{2}$, for the one with $p>4$ (Fig. 9b) and the one with $p<-4$ (Fig. 9c), respectively, account only for $8 \%$ of the total initial conditions, $4 \%$ each. The rest of the initial conditions, that is $44.4 \%$ of ICs, are attracted to the chaotic attractor (Fig. 9d). 


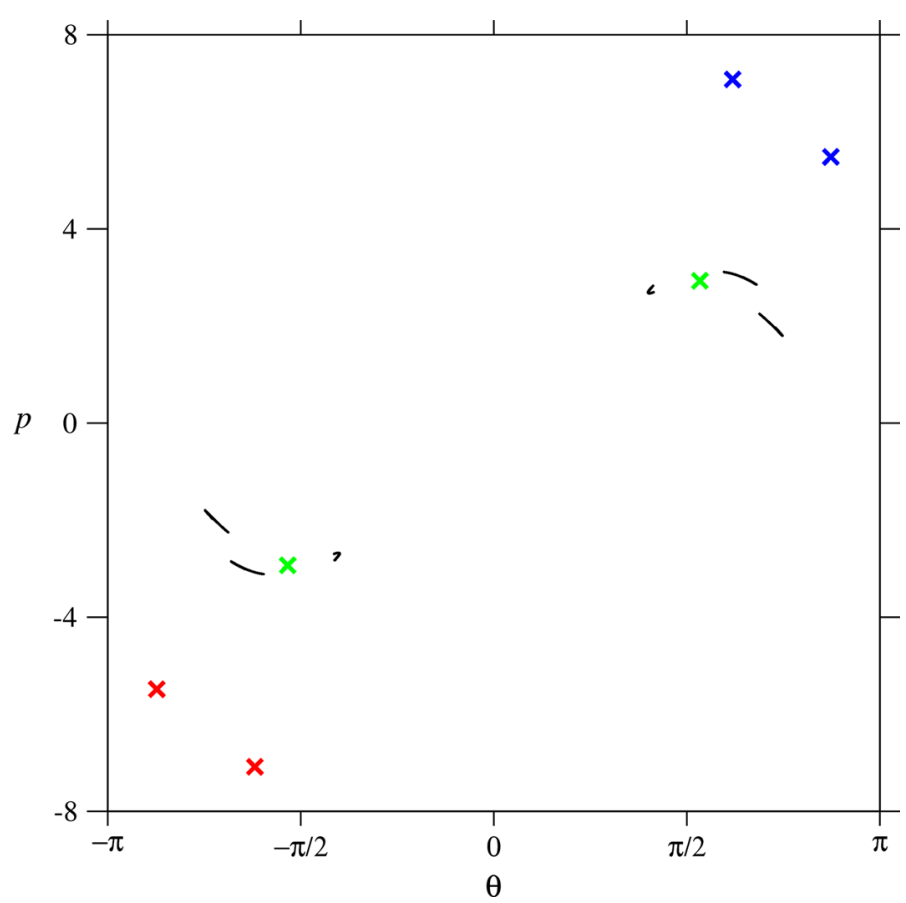

Fig. 8 Chaotic attractor (black dots) and three period-2 stable orbits for $f_{0}=4.5$ and $v=0.47330$

When these small chaotic attractors collide with the stable manifold of an UPO at the boundary of their basin of attraction, a boundary crises occurs, that is, the attractors "disappear", with a non-attractive chaotic set persisting where the attractor was before.

Finally, continuing the biparametric inspection, for very large $f_{0}$ a large chaotic attractor appears soon after dissipation is introduced. Figure 10 shows a bifurcation diagram for $f_{0}=7.5$ with $v \in(0,1)$. In this case, the bifurcation diagram was built by iterating a grid of initial conditions in the trapping region for each value of $v$ and plotting the last points of the trajectories after discarding a long transient of $4.99 \times 10^{4}$. In Fig. 10, the insets show details of a period-4 periodic window, that is, a region in parameter space for which stable periodic behavior is found.

\section{Characterization of abrupt phenomena involving chaotic attractors}

Crises are means by which the phase space of a system is globally transformed (Grebogi et al. 1987; Robert et al. 2000). In this section we describe three global bifurcations, namely a boundary crisis, a merging crisis, and an interior crisis. The cases analyzed are representative of the dynamical behavior in the biparametric plane.

Boundary crises are the general mechanism that accounts for the "vanishment" of small multi-banded chaotic attractors that exist in the case of moderate dissipation and moderate forcing, as stated before.

For example, the multi-banded chaotic attractor shown in Fig. 8 with $f_{0}=4.5$ and $v=$ 0.47330 disappears when it collides with the stable manifold of an UPO with period multiple 

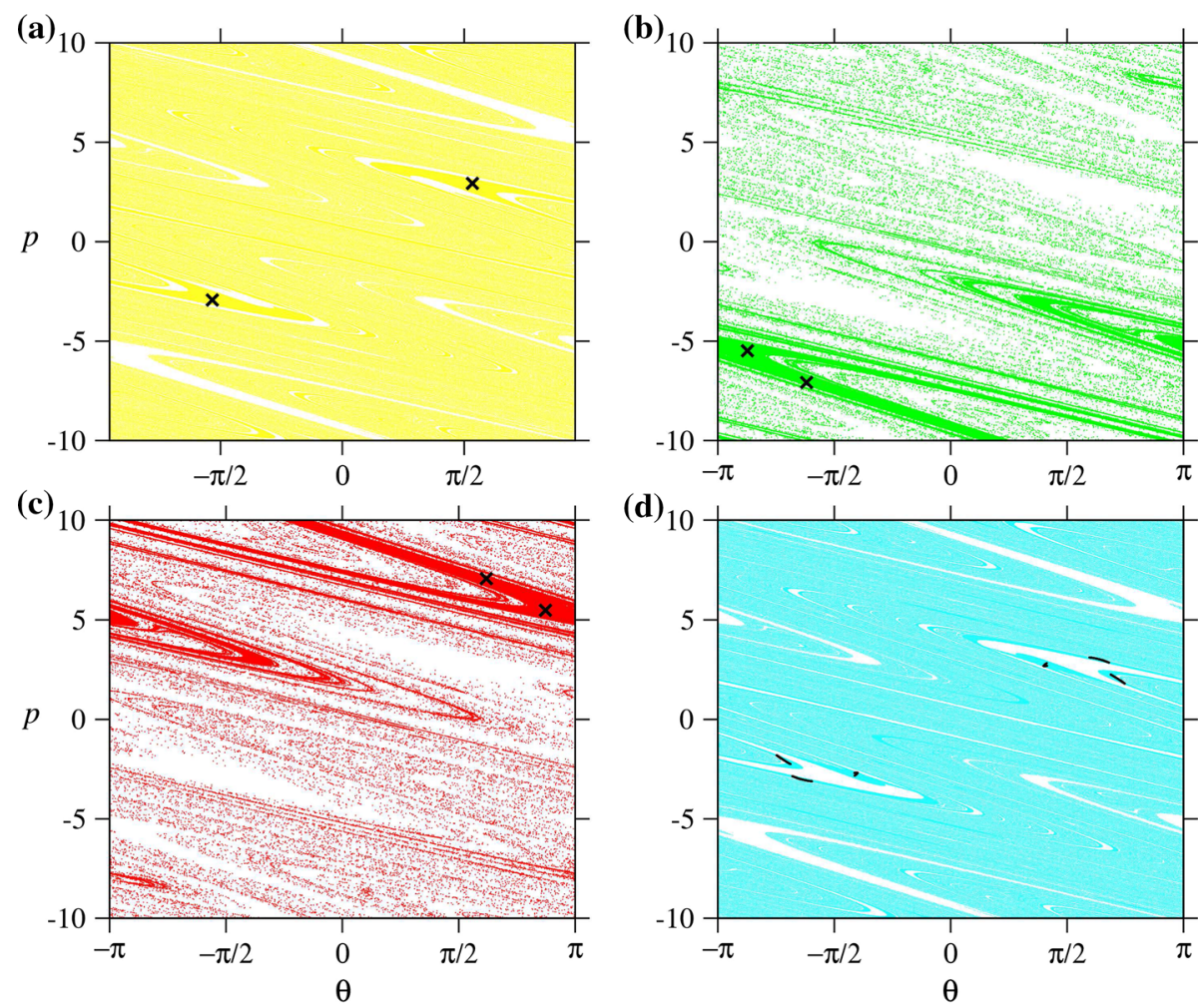

Fig. 9 Basins of attraction of the 3 period-2 attractors and of the chaotic attractor which coexist for $f_{0}=4.5$ and $v=0.47330$. Each frame shows the basin of attraction of one attractor (shown in black). Namely, a $P_{1}^{2}$, b $P_{-2}^{2}, \mathbf{c} P_{+2}^{2}$, and $\mathbf{d}$ the chaotic attractor

of six at the boundary of its basin of attraction, when $f_{0}=4.5$ and $v_{\mathrm{BC}} \approx 0.47333695$. At the crisis, the attractor is at the closure of one of the branches of the unstable manifold of the mediating UPO (Robert et al. 2000). More specifically, at the crisis, each one of the six bands of the chaotic attractor collides simultaneously with a branch of the stable manifold of the mediating UPO.

As seen in Sect. 3, before the crisis three periodic attractors coexist with the chaotic attractor. From $v=0.47330$ to $v_{\mathrm{BC}}$, the size and position of the chaotic attractor remain practically unchanged. Also the positions of the periodic attractors remain nearly constant in the phase space. On the other hand, the number of iterations that trajectories in each basin require to reach the corresponding asymptotic states changes depending on the proximity to the hyperbolic structure at the boundary.

Figure 11 presents the time needed to reach the main asymptotic states before and after the boundary crisis. The significative changes observed are evidence of the fact that, after the boundary crisis, the chaotic attractor disappears and is replaced by long chaotic transients that occur due to a chaotic saddle.

Considering the same grid used to generate the basins of Fig. 9, Fig. 11a shows the number of iterations that the trajectories in the basin of attraction of $P_{1}^{2}$ need to reach the steady state when $v=0.47330$ and $f_{0}=4.5$. In the abscissa, $k / N_{\text {tot }}$ denotes the normalized index of 


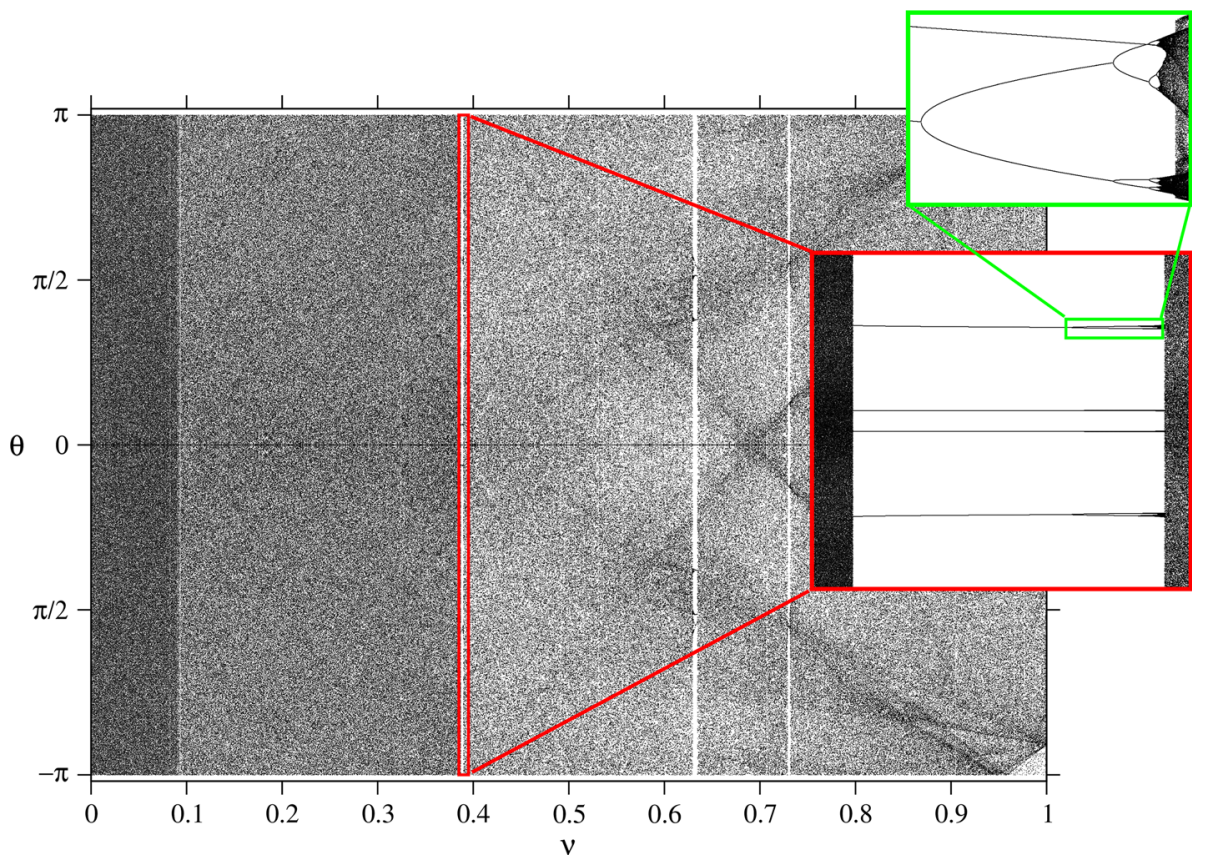

Fig. 10 Bifurcation diagram for $f_{0}=7.5$ and $v \in(0,1)$

the initial conditions in the basin of attraction of $P_{1}^{2}$, that is $N_{\text {tot }}$ is the number of initial conditions in the grid that go to $P_{1}^{2}$, and $k=1,2, \ldots, N_{\text {tot }}$ is the index of each initial condition in the basin. Fig. 11b shows the same for the chaotic attractor at the same pair of parameters. Typically, the number of iterations needed to arrive at the chaotic attractor is only slightly larger than the number of iterations needed to reach $P_{1}^{2}$. For $P_{+2}^{2}$ and $P_{-2}^{2}$ the behavior is similar to the case of $P_{1}^{2}$. It is worth to note that for each asymptotic state and at each parameter values, $N_{\text {tot }}$ has a different value according to the number of trajectories that go to the attractor.

Figure 11c shows that the typical times to reach $P_{1}^{2}$ increase dramatically as the trajectories remain for a large number of iterations in the surroundings of the chaotic saddle.

The periodic attractors $P_{1}^{2}, P_{+2}^{2}$, and $P_{-2}^{2}$ still exist after the crisis. For $f_{0}=4.5$ and $v=0.4733371$ the basins of attraction of $P_{+2}^{2}$ and $P_{-2}^{2}$ remain practically unchanged with respect to the case with $v=0.47330$ and still account for only about $8 \%$ of the initial conditions in the grid, that is, two symmetric basins, each of them with $4 \%$ of the initial conditions. Also the average number of iterations required for ICs in the basins of $P_{ \pm 2}^{2}$ to reach the asymptotic state remains practically unchanged.

Figure 12 shows the basins of attraction of the periodic attractors that exist after the crisis. The fractal dimension of boundaries is found to be $D_{f}=1.86$. Once again this value corresponds to the mean value obtained by computing $D_{0}$ for several lines with different values of $p_{0}$.

For moderate $f_{0}$, as $v$ increases, merging crises can be identified. In these global transformations of the phase space two chaotic attractors combine into one, creating a larger chaotic asymptotic state. Typically, the small multi-banded chaotic attractors that go through merging crises appear through period doubling cascades (PD) that may be preceded by a pitchfork bifurcation $(\mathrm{PB})$ if the original periodic attractor is invariant under the symmetry 
(a)

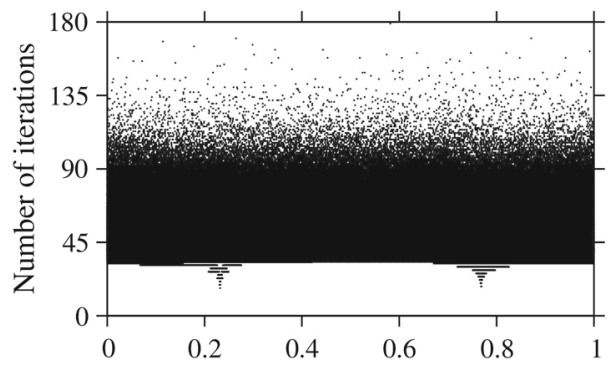

(b)

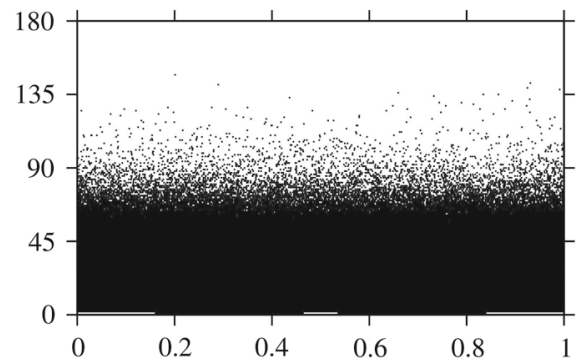

(c)

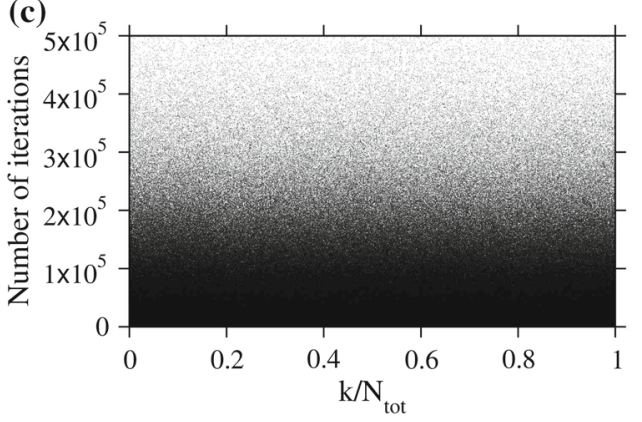

Fig. 11 Number of iterations to reach different asymptotic states, before and after the boundary crisis. a Before the crisis, for the attractor $P_{1}^{2}$ with $f_{0}=4.5$ and $v=0.47330$. b Before the crisis, for the chaotic attractor with $f_{0}=4.5$ and $v=0.47330$. c After the crisis, for the attractor $P_{1}^{2}$ with $f_{0}=4.5$ and $v=0.4733371$

transformation. The bifurcation diagram shown in Fig. 13 was obtained varying $v$ and keeping $f_{0}=4.5$ constant, starting at a stable period- 2 solution that exists when $v=0.5$. First, when $v_{P B} \approx 0.5657$, a pitchfork bifurcation gives rise to two symmetric period-2 attractors that undergo period doubling cascades leading to the formation of two double-banded chaotic attractors.

Then, as $v$ increases, both chaotic attractors grow until $v_{M C} \approx 0.676935$ when they simultaneously collide with a period-2 UPO at the boundary of their basins of attraction. At this merging crisis (MC) they become a single double-banded attractive invariant set. Figure 14 shows the elements of the merging crisis. Fig. 14a presents the two double-banded chaotic attractors and the period-2 UPO that is at the boundary that separates their basins of attraction, along with its stable manifold for the pair of values $v=0.676930$ and $f_{0}=4.5$. Fig. 14b shows the double-banded chaotic attractor that results from the merging crisis, for $v=0.676935$ and $f_{0}=4.5$.

The double-banded chaotic attractor coexists with other invariant sets. When $v_{I C} \approx$ 0.6945 , the stable manifold of an invariant chaotic saddle and the double-banded attractor collide in an interior crisis (IC). At this crisis, the size of the attractor suddenly increases and the saddle becomes part of the attractor, which also fills the gaps of the non-attractive chaotic invariant set. Fig. 15a shows the double-banded chaotic attractor and chaotic saddle before the interior crisis for $v=0.6940$, while Fig. $15 \mathrm{~b}$ presents the large single-banded attractor after the crisis for $v=0.6950$, both with $f_{0}=4.5$.

The interior crisis can be seen in terms of the collision of the double-banded chaotic attractor with the stable manifold of the unstable fixed point at the origin, which is one of 


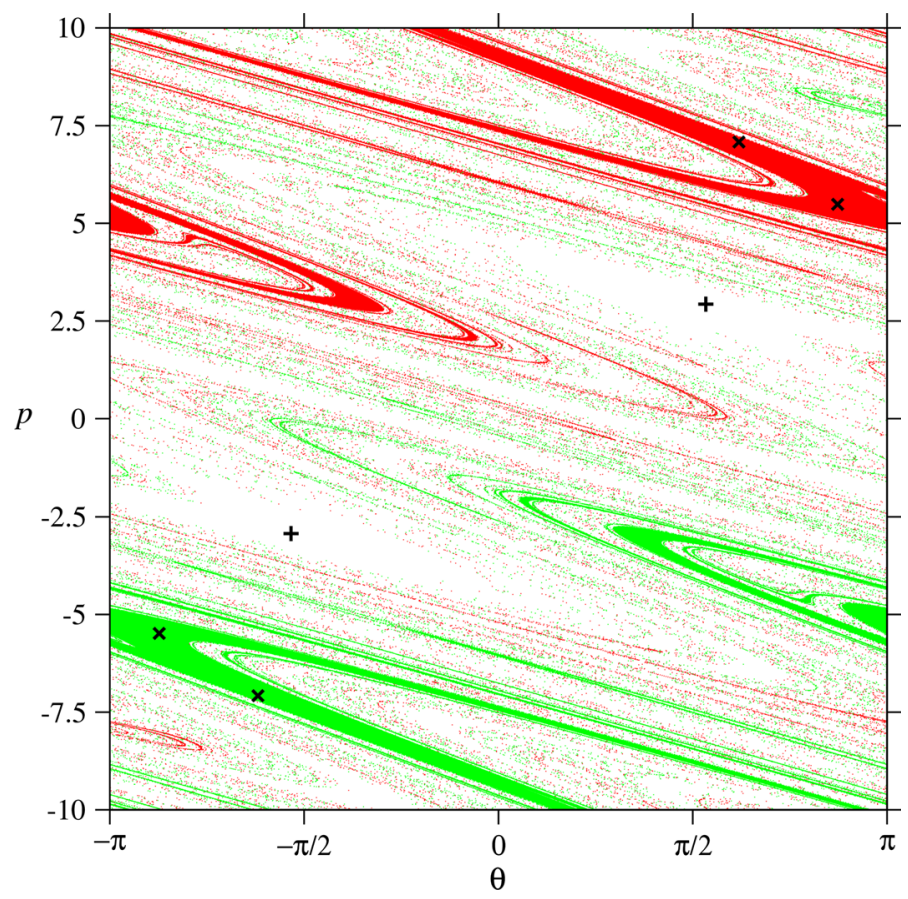

Fig. 12 After the boundary crisis: the three period- 2 attractors that coexist for $f_{0}=4.5$ and $v=0.4733371$, along with the correspondent basins of attraction (red for $P_{+2}^{2}$, green for $P_{-2}^{2}$, and white for $P_{1}^{2}$ ) (colour figure online)

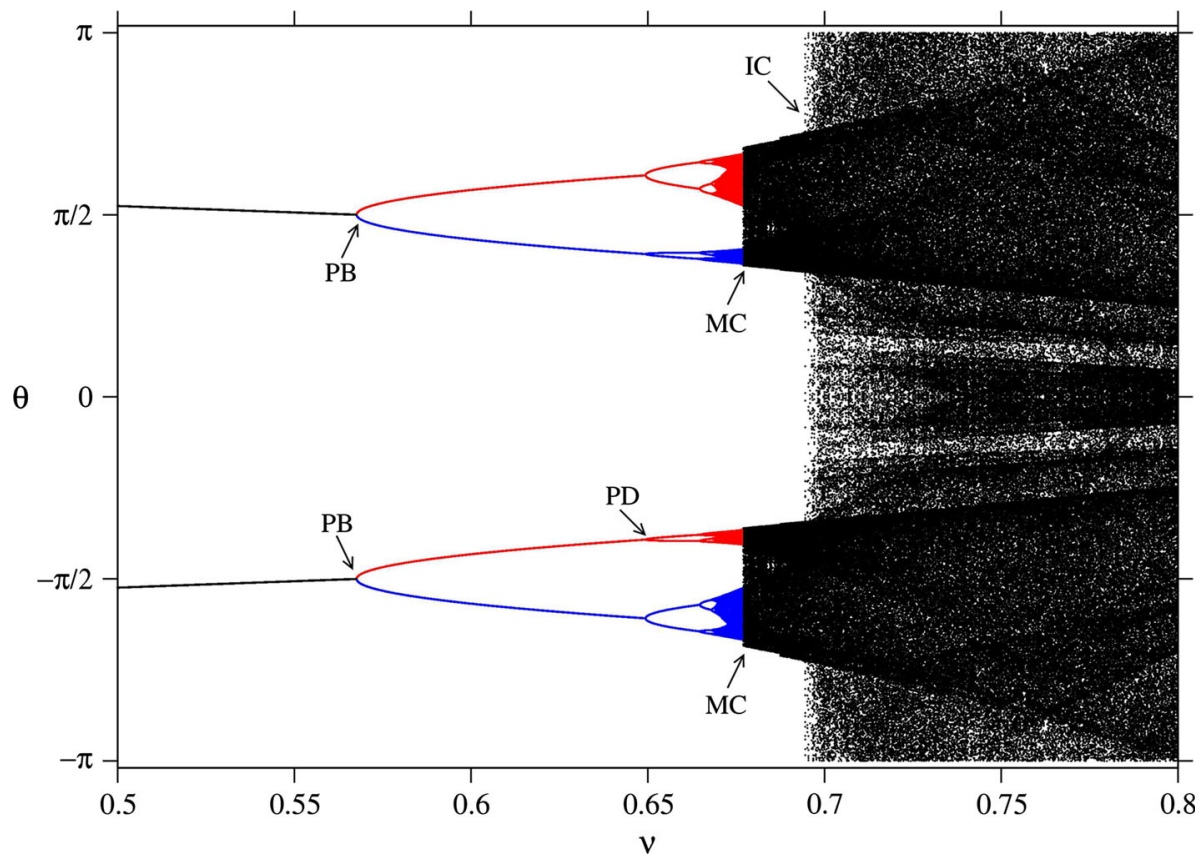

Fig. 13 Bifurcation diagram for $f_{0}=4.5$ and $v \in(0.5,0.8)$ 

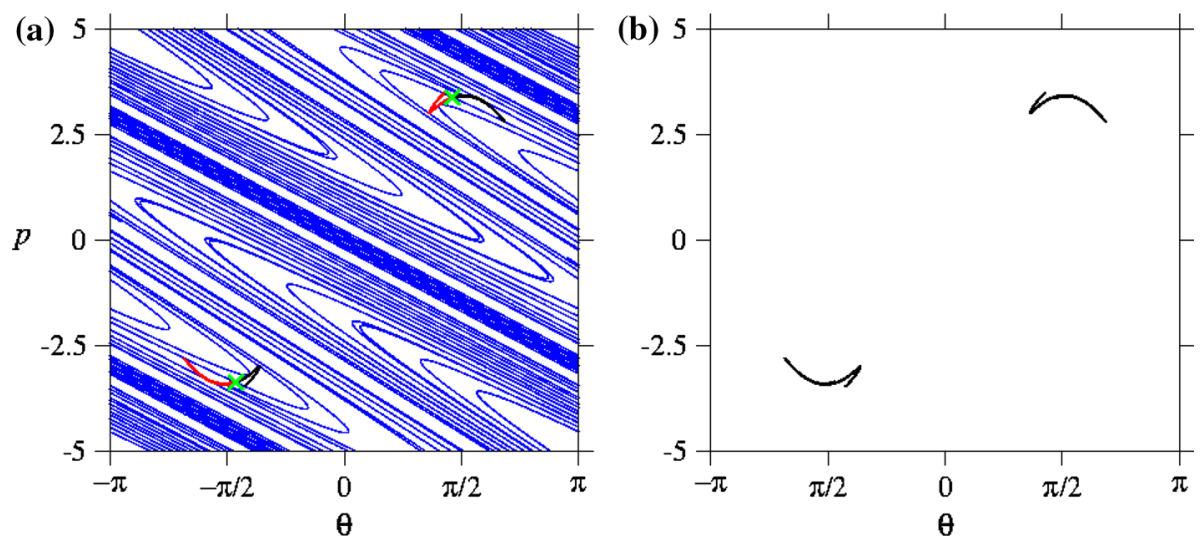

Fig. 14 Relevant dynamical elements before and after the merging crisis. a Two double-banded chaotic attractors (black and red), the period-2 UPO (green) at the boundary of their basins of attraction and the stable manifold of the UPO (blue) for $v=0.676930$ and $f_{0}=4.5$. b Double-banded attractor after the merging crisis for $v=0.676935$ and $f_{0}=4.5$ (colour figure online)
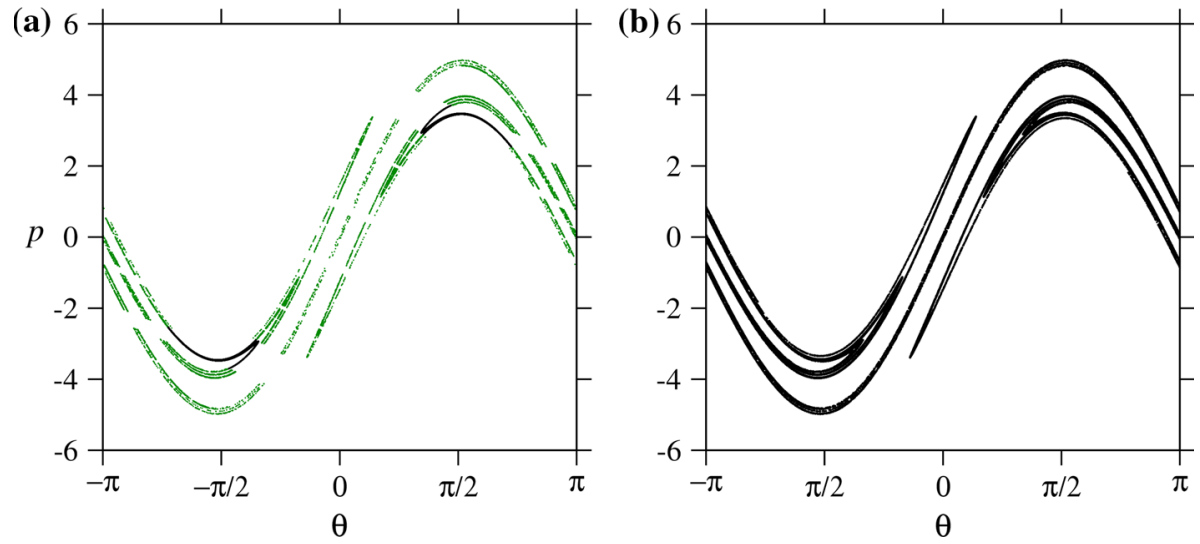

Fig. 15 a Double-banded chaotic attractor (black) and chaotic saddle (green) before the interior crisis. b Large single-banded attractor after the interior crisis (colour figure online)

the infinite UPOs embedded in the non-attractive chaotic invariant set. The fixed point at the origin was computed using a Newton method and the numerical approximation of its stable manifold was obtained by iterating the map starting with initial conditions along the stable eigendirection of the solution. At the moment of the crisis the attractor collides with the stable manifold of the UPO and is at the closure of its unstable manifold.

As general remarks, it is interesting to note that the pair of period-2 attractive solutions depicted by blue and red lines between PD and PB in the diagram of Fig. 13 indeed originates from a pair of center solutions that already exist in the Hamiltonian case, as shown by Fig. 5c, $\mathrm{d}$ (and Fig. 1e). On the other hand, the bifurcation diagram of Fig. 13 shows the sequence of dynamical phenomena that leads these period-2 attractive solutions to evolve into a large chaotic attractor that dominates the whole phase space dynamics. So, this provides us with a interesting scenario (among many) between the area preserving case to the highly dissipative case, along which some dynamical structures of the area preserving map derive solutions that are relevant in the dissipative regime. 


\section{Final remarks and conclusions}

We have performed a biparametric investigation of the multistable behavior of the general standard map. For that, we depart from the area-preserving case and inspect relevant dynamical aspects involving bifurcations, crises, and hyperbolic structures, as dissipation is introduced and varied.

For low $v$ and moderate to high $f_{0}$ the phase space is characterized by the presence of a multitude of periodic attractors. Their basins are highly interwoven and the basin boundaries are fractal. Therefore, predictability of final state is an important issue. As dissipation increases, for moderate $f_{0}$, roughly three qualitative scenarios can be found in parameter space, namely, (i) only periodic attractors of low-period, (ii) or small multi-banded chaotic attractor coexisting with a small number of low-periodic attractors, or, after several global bifurcations, (iii) large chaotic attractors. However, for high forcing parameters, large chaotic attractors are found even for low dissipation.

Besides that, we have numerically followed and characterized a boundary crisis, as well as a merging and an interior crises, depicting the role played by fundamental invariant structures for the case of intermediate $f_{0}$. Specifically, unstable periodic orbits and their hyperbolic invariant manifolds are central in the mechanisms by which the phase space is globally transformed.

We emphasize also that some dynamical invariant sets present in the area-preserving map play a central role as dissipation varies and bifurcations occur. Specifically, in the case of the merging and the interior crises last reported, both the stable period-two solutions from which a sequence of bifurcations originates the chaotic attractor and the unstable periodic orbit embedded at the chaotic saddle to which the attractor collides are solutions that already exist or that bifurcated from solutions of the Hamiltonian case.

The numerical study performed and the characterization of the dynamical invariant sets involved in the global transformations of the phase space is important to the comprehension of multistable systems and the observed phenomenology is common to a wide variety of dynamical systems.

Acknowledgements This work was supported by FAPESP (Brazil) Grants 2013/07174-4 and 2015/13339-1 and by CAPES (Brazil).

\section{References}

Aguirre J, Vallejo JC, Sanjuán MAF (2001) Wada basins and chaotic invariant sets in the Hénon-Heiles system. Phys. Rev. E 64:066208. https://doi.org/10.1103/PhysRevE.64.066208

Berretti A, Chierchia L (1990) On the complex analytic structure of the golden invariant curve for the standard map. Nonlinearity 3:39-44. https://doi.org/10.1088/0951-7715/3/1/003

Calleja R, Celletti A (2010) Breakdown of invariant attractors for the dissipative standard map. Chaos 20:013121. https://doi.org/10.1063/1.3335408

Calleja R, Figueras JL (2012) Collision of invariant bundles of quasi-periodic attractors in the dissipative standard map. Chaos 22:033114. https://doi.org/10.1063/1.4737205

Celletti A, Della Penna G, Froeschlé C (1998) Analytical approximation of the solution of the dissipative standard map. Int. J. Bifurc. Chaos 8:2471-2479. https://doi.org/10.1017/S0252921100073127

Celletti A, Di Ruzza S (2011) Periodic and quasi-periodic orbits of the dissipative standard map. Discrete Contin. Dyn. Syst. Ser. B 16:151-171. https://doi.org/10.3934/dcdsb.2011.16.151

Chirikov BV (1979) A universal instability of many-dimensional oscillator systems. Phys. Rep. 52(5):263-379. https://doi.org/10.1016/0370-1573(79)90023-1

de Assis SC, Terra MO (2014) Escape dynamics and fractal basin boundaries in the planar earthmoon system. Celest. Mech. Dyn. Astron. 120(2):105-130. https://doi.org/10.1007/s10569-014-9567-2 
Feudel U, Grebogi C (2003) Why are chaotic attractors rare in multistable systems? Phys. Rev. Lett. 91:134102. https://doi.org/10.1103/PhysRevLett.91.134102

Feudel U, Grebogi C, Hunt BR, Yorke JA (1996) Map with more than 100 coexisting low-period periodic attractors. Phys. Rev. E 54:71-81. https://doi.org/10.1103/PhysRevE.54.71

Gelfreich VG (1999) A proof of the exponentially small transversality of the separatrices for the standard map. Commun. Math. Phys. 201:155-216. https://doi.org/10.1007/s002200050553

Grebogi C, Ott E, Yorke JA (1987) Chaos, strange attractors, and fractal basin boundaries in nonlinear dynamics. Sci. New Ser. 238(4827):632-638

Greene JM (1979) A method for determining a stochastic transition. J. Math. Phys. 20(6):1183-1201. https:// doi.org/10.1063/1.524170

Martins LC, Gallas JAC (2008) Multistability, phase diagrams and statistical properties of the kicked rotor: a map with many coexisting attractors. Int. J. Bifurc. Chaos 18(06):1705-1717. https://doi.org/10.1142/ S0218127408021294

Miguel N, Simó C, Vieiro A (2013) From the Henón conservative map to the chirikov standard map for large parameter values. Regul. Chaot. Dyn. 18:469-489. https://doi.org/10.1134/S1560354713050018

Miguel N, Simó C, Vieiro A (2015) Effect of islands in diffusive properties of the standard map for large parameter values. Found. Comput. Math. 15:89-123. https://doi.org/10.1007/s10208-014-9210-3

Ott E (1993) Chaos in Dynamical Systems. Cambridge University Press, Cambridge

Robert C, Alligood KT, Ott E, Yorke JA (2000) Explosions of chaotic sets. Phys. D Nonlinear Phenom. 144(1-2):44-61. https://doi.org/10.1016/S0167-2789(00)00074-9

Tirnakli U, Borges EP (2016) The standard map: from Boltzmann-Gibbs statistics to Tsallis statistics. Sci. Rep. 6:23644. https://doi.org/10.1038/srep23644 